\title{
SARS-CoV-2 infection in children with nephrotic syndrome
}

\author{
Sudarsan Krishnasamy ${ }^{1} \cdot$ Aditi Sinha $^{1}$ (1) $\cdot$ Arvind Bagga $^{1}$ (i)
}

Received: 22 November 2021 / Revised: 22 November 2021 / Accepted: 22 November 2021 / Published online: 10 January 2022

(c) The Author(s), under exclusive licence to International Pediatric Nephrology Association 2022

\section{Dear Editors,}

We read with interest the published systematic review on COVID-19 in 43 patients with idiopathic nephrotic syndrome [1]. The authors report that the disease is usually mild, uncommonly requires respiratory support, and is associated with favorable outcomes regardless of concomitant immunosuppression. In 5 patients, SARS-CoV2 infection was complicated by a relapse that showed satisfactory response to therapy with prednisolone. Two children had onset of the illness following the infection, and it was speculated whether the virus might have precipitated the occurrence of the disease. Based on their findings, the authors propose guidelines on the management of children with nephrotic syndrome, including advice on immunosuppression, monitoring of disease, and advice regarding vaccination against COVID-19. We had recently published our experience on a similar number of patients with nephrotic syndrome and COVID-19, one month beyond the database searches run by the authors. Since these were not included in the review, we wish to emphasize new findings in context of the systematic review.

During 2020-2021, we diagnosed SARS-CoV2 infection in 88 children with chronic kidney diseases in four tertiary care centers in New Delhi, including 44 patients with nephrotic syndrome [2]. Twenty-seven $(61.4 \%)$ patients had steroid sensitive disease; over one-half $(24,54.5 \%)$ presented in relapse, including 2 patients with the initial episode of nephrotic syndrome. The majority of the patients $(32,72.7 \%)$ were receiving immunosuppressive medications, chiefly prednisolone, calcineurin inhibitor, or mycophenolate mofetil. While $93 \%$ of patients had asymptomatic or mild COVID-19 at presentation, 10 (22.7\%) developed moderate to severe COVID-19 during hospital stay, with $7(15 \%)$ requiring non-invasive or mechanical ventilation,

Aditi Sinha

aditisinhaaiims@gmail.com

1 Division of Nephology, Department of Pediatrics, All India Institute of Medical Sciences, Ansari Nagar, New Delhi 110029, India and $3(6.8 \%)$ needing vasopressor support. Nine patients with nephrotic syndrome had acute kidney injury (AKI) at presentation, and 3 developed AKI later. Overall, 5 (11.3\%) patients developed stage 3 AKI [3] that required kidney replacement therapy. Two (4.5\%) patients, both with steroid resistant nephrotic syndrome who presented during a relapse, succumbed to respiratory failure and shock. Compared to patients with nephrotic syndrome in remission or with other kidney diseases, patients with nephrotic syndrome in relapse showed a six-fold risk of developing severe complications during COVID-19, such as severe AKI (stages 2-3), shock, respiratory failure, encephalopathy, or death. Immunosuppression was not associated with adverse outcomes.

Contrary to the findings of the systematic review, our findings indicate the need for cautious monitoring of patients with nephrotic syndrome with disease relapse and COVID19 . We propose that the altered hemodynamic state in relapse, including risks associated with hypovolemia, interstitial edema, and/or use of diuretics, ACE-inhibitors, and other nephrotoxic medications, may contribute to a higher risk of complications associated with COVID-19. The 2 patients who died received appropriate intensive care, and mortality was certainly not due to lack of resources.

Data from the systematic review and our case series suggest that a significant proportion of patients with nephrotic syndrome with SARS-CoV2 infection present during a relapse. Patients with moderate to severe COVID-19 require reduction of high-dose corticosteroid therapy and monitoring for complications, including cardiorespiratory failure and AKI. Patients in remission, whether on or off immunosuppression, may be managed satisfactorily with the usual standard of care.

\section{Declarations}

Competing interests The authors declare no competing interests. 


\section{References}

1. Morello W, Vianello FA, Proverbio E, Peruzzi L, Pasini A, Montini G (2021) COVID-19 and idiopathic nephrotic syndrome in children: systematic review of the literature and recommendations from a highly affected area. Pediatr Nephrol. https://doi.org/10. 1007/s00467-021-05330-2

2. Krishnasamy S, Mantan M, Mishra K, Kapoor K, Brijwal M, Kumar M, Sharma S, Swarnim S, Gaind R, Khandelwal P, Hari P, Sinha A, Bagga A (2021) SARS-CoV-2 infection in children with chronic kidney disease. Pediatr Nephrol. https://doi.org/10. 1007/s00467-021-05218-1
3. Lameire NH, Bagga A, Cruz D, De Maeseneer J, Endre Z, Kellum JA, Liu KD, Mehta RL, Pannu N, Van Biesen W, Vanholder R (2013) Acute kidney injury: an increasing global concern. Lancet 382:170-179

Publisher's note Springer Nature remains neutral with regard to jurisdictional claims in published maps and institutional affiliations. 